NASA

Technical Memorandum 105847
AVSCOM

Technical Report 92-C-023

\title{
Direct Numerical Simulation of Instabilities in Parallel Flow With Spherical Roughness Elements
}

R.G. De Anna

Propulsion Directorate

U.S. Army Aviation Systems Command

Lewis Research Center

Cleveland, Ohio 


\title{
DIRECT NUMERICAL SIMULATION OF INSTABILITIES IN PARALLEL FLOW WITH SPHERICAL ROUGHNESS ELEMENTS
}

\author{
R. G. De Anna
}

\author{
U.S. Army (AVSCOM) Propulsion Directorate \\ NASA Lewis Research Center \\ Cleveland, Ohio 44135
}

\begin{abstract}
Results from a direct numerical simulation of laminar flow over a flat surface with spherical roughness elements using a spectralelement method are given. The numerical simulation approximates roughness as a cellular pattern of identical spheres protruding from a smooth wall. Periodic boundary conditions on the domain's horizontal faces simulate an infinite array of roughness elements extending in the streamwise and spanwise directions, implies the parallel-flow assumption, and results in a closed domain. A body force, designed to yield the horizontal Blasius velocity in the absence of roughness, sustains the flow. Instabilities above a critical Reynolds number reveal negligible oscillations in the recirculation regions behind each sphere and in the free stream, high-amplitude oscillations in the layer directly above the spheres, and a mean profile with an inflection point near the sphere's crest. The inflection point yields an unstable layer above the roughness (where $U^{\prime \prime}(y)<0$ ) and a stable region within the roughness (where $U^{\prime \prime}(y)>0$ ). Evidently, the instability begins when the low-momentum or wake region behind an element, being the region most affected by disturbances (purely numerical in this case), goes unstable and moves. In incompressible flow with periodic boundaries, this motion sends disturbances to all regions of the domain. In the unstable layer just above the inflection point, the disturbances grow while being carried downstream with a propagation speed equal to the local mean velocity; they do not grow amid the low energy region near the roughness patch. The most amplified disturbance eventually arrives at the next roughness element downstream, perturbing its wake and inducing a global response at a frequency governed by the streamwise spacing between spheres and the mean velocity of the most amplified layer.
\end{abstract}




\section{Introduction}

Generally, surface roughness promotes transition in the sense that under otherwise identical conditions, transition occurs at a lower Reynolds number on a rough wall than on a smooth one [Dryden, 1953, Sedney, 1973]. Evidently, roughness elements give rise to additional disturbances which add to those already present in the laminar, boundary layer. A sufficiently rough surface may advance the transition location upstream of the smooth-wall position; otherwise, extended regions of relatively steady but distorted, laminar flow may appear [Sedney, 1973]. Roughness beginning downstream of the location where linear disturbances grow may enhance growth in TollmienSchlichting $(T S)$ waves [Corke \& Others, 1986], while disturbances from roughness beginning further upstream may "bypass" the known linear instability processes [Reshotko \& Leventhal, 1981].

\subsection{Isolated roughness}

The characteristics of flow over three-dimensional roughness elements depend on the interaction between the natural boundary-layer vorticity and the obstacle [Mason \& Morton, 1987]. Upon encountering a three-dimensional surface protuberance, the vortex lines may concentrate to form a visible vortex core which trails downstream. Experiments at low velocity reveal a single smoke streak trailing downstream from the element [Mochizuki, 1961]. At higher velocities, a horseshoe vortex and a pair of smoke spirals (chimney vortices fixed in space and due to the fluid entering into the rear separation pocket) appear close behind the sphere forming two parallel smoke filaments trailing downstream parallel to the wall (called the trailing vortex). As the velocity increases, the trailing vortex filaments begin "waving," while growing over some distance downstream. Further downstream, they cease to grow after oblique stretching by the main stream's shearing stresses. At higher wind speed these vortices shed at a dimensionless frequency (non-dimensionalized as a Strouhal number, $\left.S t_{k}=f k / U_{k}\right)$ ranging from 0.1 to 0.4 depending on Reynolds number [Gupta, 1980, Gregory \& Walker, 1951]. The trailing vortex filaments show waviness at $R e_{k}=U_{k} k / \nu=350$ and shedding at $R e_{k}=700$ [Mochizuki, 1961]. (The undisturbed velocity at the crest of the roughness equals $U_{k}$, and the roughness height equals 
$k$.) In all cases, the dimensionless instability frequency, $f \nu / U_{\infty}^{2}$, falls above the unstable region of the stability diagram for $T S$ waves.

\subsection{Distributed roughness}

In contrast to the well understood sequence of instabilities leading to transition around isolated roughness, the process for distributed roughness remains unknown [Morkovin, 1989]. Although some similar mechanisms may exist, the ordered behavior near single elements vanishes when neighboring elements are present. We expect an interaction between the streamwise vortices engendered by upstream elements and the vortex system of those further downstream; however, amalgamation of the wakes from upstream elements should produce a low-momentum region amid the roughness patch. This lowers the effective roughness Reynolds number, $R e_{k}$, which depends on the velocity at the elevation of the roughness in the smooth-wall boundary layer. Therefore, with equal roughness Reynolds numbers, a element in a distributed roughness patch encounters an extenuated mainstream compared to an isolated element. In this case, closely-packed elements will not beget the strong horseshoe and chimney vortices necessary to initiate the shedding process typically found with isolated elements until reaching a much higher Reynolds number.

\section{Numerical Simulation of Roughness}

Several numerical studies investigated the mean flow and disturbance field around isolated three-dimensional roughness elements [Mason \& Sykes, 1979, Mason \& Morton, 1987, Tadjfar, 1990]. Tadjfar employed a three-dimensional triple-deck with time-harmonic free-stream disturbances; and Mason et al. solved the full threedimensional, unsteady Navier-Stokes equations. The work presented here represents the first computational investigation of distributed roughness using both realistic geometry and the three-dimensional, unsteady Navier-Stokes equations.

\subsection{Distributed roughness pattern under study}

The distributed roughness under investigation consists of an array of identical spheres protruding from a flat plate according to pre- 


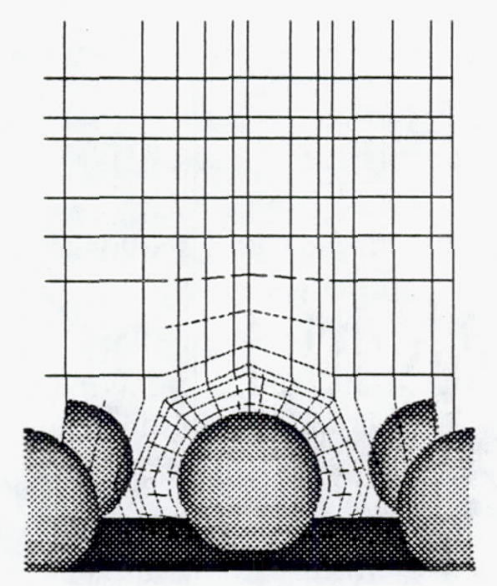

Figure 1: Side view of the standard density domain showing a $5 X 5 X 5$ mesh along the vertical symmetry plane extending from the wall to the free stream.

vious experiments [Kendall, 1981, Tadjfar \& Others, 1985]. Whereas the experimental studies consisted of a spatial array of a large number of spheres, the computational domain consists of the single cell shown in figures 1 and 2 .

\subsection{Boundary conditions}

All previous experiments with distributed roughness were done in spatially-developing boundary layers or "open" flows. By applying periodic boundary conditions to the horizontal faces of a single cell and an impermeable boundary condition along the cell's top, we simulate an infinite array of roughness elements extending upstream, downstream, and spanwise. These boundary conditions effectively "close" the domain and result in a parallel flow.

In simple geometries, periodic conditions allow us to simulate spatial development by interpreting the time-dependent data as that coming from a domain moving downstream with the group velocity of the disturbances. This "moving box" approach is often effective in these geometries [Singer \& Others, 1986, Spalart \& Yang, 1987, Laurien \& Kleiser, 1989].

This artifice fails in complex geometries, however. The domain must remain fixed at a given streamwise position, and temporal data 


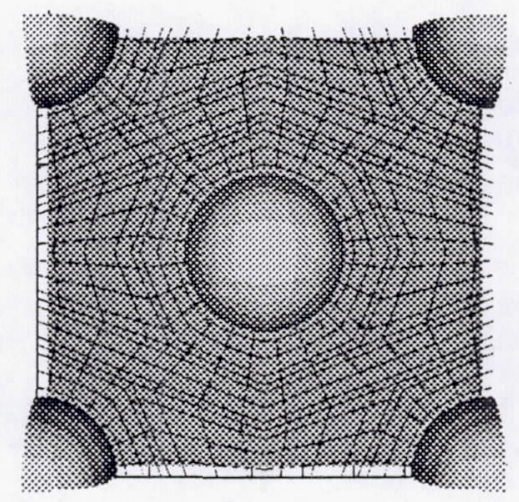

Figure 2: Plan view of the standard density domain showing a $5 X 5 X 5$ mesh along a horizontal plane passing through the center of the spheres.

can only be used to indicate frequency and phase content along with relative amplitude between signals from different locations; it cannot reveal anything about growth rates since disturbances cannot grow at a fixed location in a flow with steady mean quantities. The state with steady mean flow is called the time-asymptotic state. This restriction does, however, provide a convenient check on the numerical scheme: After achieving the time-asymptotic stage, a solution showing growing fluctuations indicates poor spatial or temporal resolution, while decaying fluctuations cannot occur. (These comments do not apply to time-asymptotic flows with imposed disturbances. In these cases, a time-asymptotic flow may be "perturbed" by introducing artificial disturbances which may grow or decay in time.)

Finally, a word about roughness and $T S$ waves is appropriate. Although experiments reveal enhanced growth in the TS band of frequencies when roughness begins downstream of the critical point for $T S$ wave growth, the periodic and free-stream boundary conditions applied to the single roughness cell shown in figure 1 preclude $T S$ waves- $T S$ waves have wavelengths which typically equal ten boundary-layer thicknesses and amplitudes which decay asymptotically with distance from the wall. Unfortunately, the finite height domain, imposed free-stream boundary conditions, and relatively short cell length are incongruous with this type wave. 


\subsection{Numerical procedure}

Both the incompressible continuity equation, given by

$$
\frac{\partial u_{j}}{\partial x_{j}}=0
$$

and the incompressible Navier-Stokes equation, given by

$$
\frac{\partial u_{i}}{\partial t}+u_{j} \frac{\partial u_{i}}{\partial x_{j}}=-\frac{1}{\rho} \frac{\partial p}{\partial x_{i}}+f_{i}+\nu \frac{\partial^{2} u_{i}}{\partial x_{j} \partial x_{j}},
$$

are solved in the domain described in sections 2.1 and 2.2 using a spectral-element code. The spectral-element method employs a three-step, time-splitting scheme where the non-linear, pressure, and viscous terms in the incompressible Navier-Stokes equations are written in separate fractional steps [Orszag \& Kells, 1980]. By introducing intermediate velocities, these steps yield consecutive solutions based first on the non-linear, then on the pressure, and finally on the viscous terms.

\subsection{Input parameters}

When the incompressible Navier-Stokes equations (see equation 2) are written in non-dimensional form, three dimensionless quantities appear: the Reynolds number, $R e=U L / \nu$; the Strouhal number, $S t=f L / U$; and the Froude number, $F_{r}=U / \sqrt{g L}$. With periodic boundary conditions, a body force generates the velocity, and a relationship exists between the Reynolds and Froude numbers. Furthermore, in a flow without external forcing, the Strouhal number governs unsteadyness due to naturally arising instabilities. Hence, the Reynolds number or, for a given geometry, the fluid viscosity and the body force, completely determines the flow.

Here we employ a special body force which, when twice differentiated with respect to $y$, yields the Blasius profile in the absence of roughness elements. It is derived by considering the streamwise momentum equation in a boundary layer on a smooth, flat surface with no imposed streamwise pressure gradient. Equation 2 in this case reduces to

$$
u \frac{\partial u}{\partial x}+v \frac{\partial u}{\partial y}=\nu \frac{\partial^{2} u}{\partial^{2} y}
$$


By interpreting the right-hand side of equation 3 as a body force, we get

$$
u \frac{\partial u}{\partial x}+v \frac{\partial u}{\partial y}=f_{B}(y)
$$

with

$$
f_{B}(y) \equiv \nu \frac{\partial^{2} u_{B}(y)}{\partial^{2} y}
$$

while the subscript $B$ refers to the Blasius value. By twice differentiating the Blasius velocity profile according to equation 5 , we obtain a body force which yields the Blasius profile on a smooth, flat surface with no imposed pressure gradient. When this body force is applied to a domain with surface roughness elements, the resulting velocity field represents the influence of the roughness elements on the Blasius boundary layer.

\subsection{Time asymptotic state}

From a given initial state, a certain time elapses while meanflow quantities develop towards a time-asymptotic state. The time required to reach this state depends on the starting solution as well as the longer of the convection and diffusion times.

The convection time to reach the asymptotic state depends on the time for a particle to pass across the domain, given by $\tau_{c}=L_{x} / U_{\infty}$, where $U_{\infty}$ equals the streamwise free-stream velocity, and $L_{x}$ equals a streamwise length scale.

The diffusion time to reach the time-asymptotic state depends on momentum diffusion across the layer. For a fixed boundary-layer thickness and viscosity, momentum diffusion across a boundary layer of thickness $\delta$ requires $\delta^{2} / \nu$ time.

The ratio of diffusion to convection times equals

$$
\frac{\tau_{d}}{\tau_{c}}=\frac{\delta^{2} / \nu}{L_{x} / U_{\infty}}=\left(\frac{\delta}{L_{x}}\right) \frac{U_{\infty} \delta}{\nu}=\left(\frac{\delta}{L_{x}}\right) R e_{\delta} .
$$

Typically, the Reynolds number equals several hundred, and the ratio of boundary-layer thickness to streamwise length is of order one; hence, diffusion requires two orders-of-magnitude more time than convection. For typical flows of interest, diffusion requires $10^{3}$ time units. Since the first-order time-accurate spectral-element 


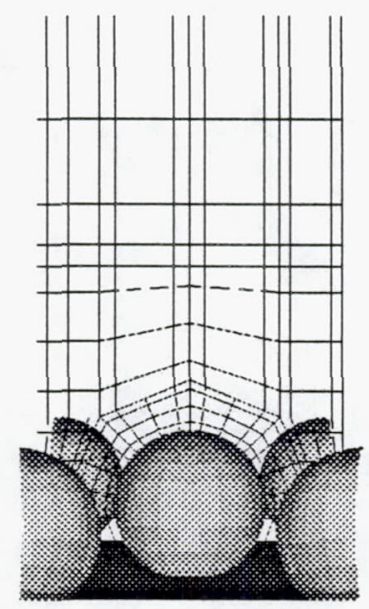

Figure 3: Side view of the high-density domain showing a $5 X 5 X 5$ mesh along the vertical symmetry plane extending from the wall to the free stream.

method requires small time steps-typically $\Delta t \leq 0.01$ - for good accuracy, approximately $10^{5}$ time steps elapse before reaching a timeasymptotic flow.

\subsection{Cases studied}

Some previous experiments using distributed roughness elements [Gartshore \& de Croos, 1979, Lee \& Soliman, 1977, Kendall, 1981] found that roughness "density," or spacing between elements, controls certain mechanisms responsible for transition. Based on this idea, I selected three different roughness densities for study: standard, low, and high. The roughness density was varied by stretching or compressing the domain in the streamwise direction while holding the spanwise dimension, the number of finite elements, and the total number of grid points constant. Consequently, the high-density domain was shorter (see figure 3), and the low-density domain was longer (see figure 4) than the standard domain. The standard-density domain consists of a square with streamwise and spanwise lengths of 8.4; the high-density domain consists of a rectangle with a streamwise length of 6.0 and spanwise length 8.4; and the low-density domain has a streamwise length of 12.0 and a spanwise length of 8.4. All cases used $R e_{k}=175, k / \delta^{*}=0.72$, and the Blasius based body force 


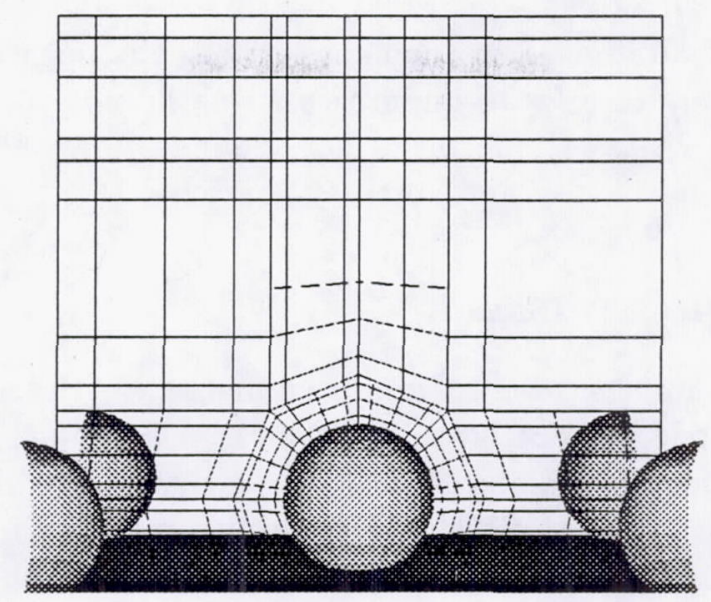

Figure 4: Side view of the low-density domain showing a $5 X 5 X 5$ mesh along the vertical symmetry plane extending from the wall to the free stream.

(see section 2.4) to drive the flow. For each case, the one-cell grid consisted of 144 elements and a $5 X 5 X 5$ mesh within each element. The standard-density case was also run with a finer, $7 X 7 X 7$, mesh.

The calculations performed on a single-cell domain correspond to the assumption of "strong periodicity" [Karniadakis \& Others, 1988]. Unfortunately, we have no a priori knowledge whether or not a given flow will behave differently when the number of cells are changed. To gain insight into the effect of the streamwise, periodic boundary conditions on the nature of the disturbance wavelength and frequency, a two-cell high-density case and a two-cell low-density case were run. These two-cell cases contained two 144 -element grids aligned in the streamwise direction, used the $5 X 5 X 5$ mesh, and were twice as long as their single-cell counterparts. A change in results between the one- and two-cell cases will indicate the presence of a sub-harmonic disturbance.

\section{Steady State Results}

Since we compute the unsteady Navier-Stokes equations until a time-asymptotic state is reached, all of the results are unsteady above a critical Reynolds number. To distinguish steady results from 
unsteady results (given in the next section) the term "steady state" is used loosely to refer to quantities which do not vary significantly with time. These include mean flow boundary-layer quantities and instantaneous velocity and vorticity contours.

\subsection{Mean flow results}

Results for the three different roughness densities using a $5 X 5 X 5$ mesh with one cell are given in table 1. Also appearing in table 1 are results for the standard-density case using a $7 X 7 X 7$ mesh. The corresponding smooth-wall, Blasius results are given for comparison. Some of the quantities appearing in the table need to be defined before discusing the results. The ratio of rough-wall to smooth-wall friction coefficients equals $\frac{\left(C_{f}\right)_{r}}{\left(C_{f}\right)_{s}}$. The maximum velocity divergence, equals $(\nabla \cdot V)_{m}$, and the velocity divergence integrated over the entire domain, equals $(\nabla \cdot V)_{a}$-both indicate the quality of the numerical solution, since they equal zero in incompressible flow. Finally, the displacement and momentum thicknesses appear here in dimensionless form.

According to table 1 , an improvement in grid resolution from a $5 X 5 X 5$ to a $7 X 7 X 7$ mesh per element yields a slight increase in drag, evidence that the course mesh fails to capture the smaller scales required to resolve the flow. The results also show an increase in $\delta^{*}$ and a decrease in $\theta$ compared to the smooth-wall (Blasius) values and consistent with inflected or adverse pressure-gradient profiles.

\begin{tabular}{|c|c|c|c|c|c|c|c|}
\hline density & mesh & $\delta^{*}$ & $\theta$ & $H$ & $(\nabla \cdot V)_{m}$ & $(\nabla \cdot V)_{a}$ & $\frac{\left(C_{f}\right)_{r}}{\left(C_{f}\right)_{s}}$ \\
\hline stand. & 5X5X5 & 1.85 & .508 & 3.64 & 0.0200 & -0.0176 & 2.62 \\
stand. & 7X7X7 & 1.88 & .530 & 3.57 & - & - & 2.64 \\
high & 5X5X5 & 1.80 & .473 & 3.80 & 0.0148 & -0.0128 & 2.69 \\
low & 5X5X5 & 1.84 & .529 & 3.48 & 0.0322 & -0.0352 & 2.49 \\
Blas. & theory & 1.72 & .664 & 2.59 & 0.0 & 0.0 & 1.0 \\
\hline
\end{tabular}

Table 1: Mean flow results for the various roughness densities and the corresponding smooth-wall, Blasius data

Compared to the standard domain, the high-density case effects an increase in $\frac{\left(C_{f}\right)_{r}}{\left(C_{f}\right)_{s}}$ and $H$ and a decrease in both $\delta^{*}$ and $\theta$, the 


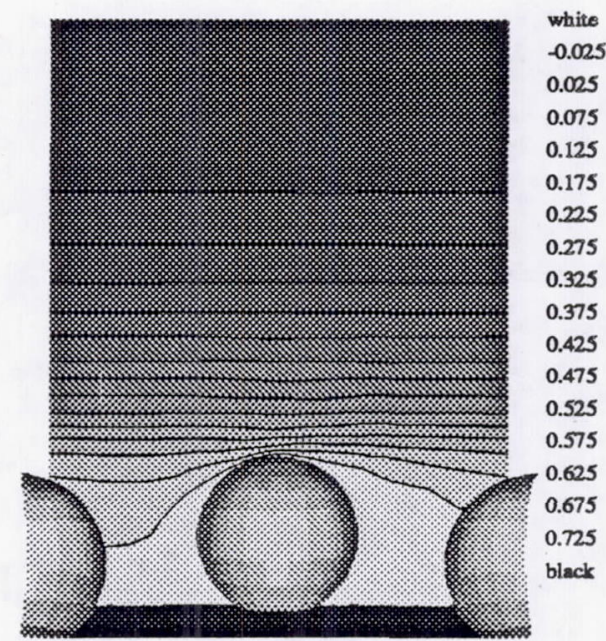

Figure 5: Side view of the streamwise velocity contours on a standard-density domain with a $5 X 5 X 5$ mesh along the vertical symmetry plane extending from the wall to the free stream.

opposite occurs in the low-density case.

\subsection{Velocity contours standard density domain}

The standard-density domain velocity contours are given in figures 5 through 10 ; vorticity contours are given in figure 11 . Figures 5 and 6 show the streamwise and vertical velocity on a plane through the center of the middle sphere. A small reverse flow appears in the region behind the sphere. The vertical velocity (figure 6 ) equals zero above two roughness heights while remaining within 4.4 percent of the free-stream velocity near the elements. The small upward velocity behind the element remains confined to the region between the centerline and crest. At this Reynolds number, the sign and location accede with the chimney vortex of an isolated roughness elementa vortex rotating counter to and above the horseshoe vortex. In this case, however, the neighboring roughness elements beget a lowmomentum region and prevent the formation of robust horseshoe and chimney vortices. Instead, a flow dominated by kinematics results: continuity in concert with the solid-wall boundary conditions. The upward velocity behind the sphere depends on continuity: the fluid passing around the sides of the element converges in the wake and 


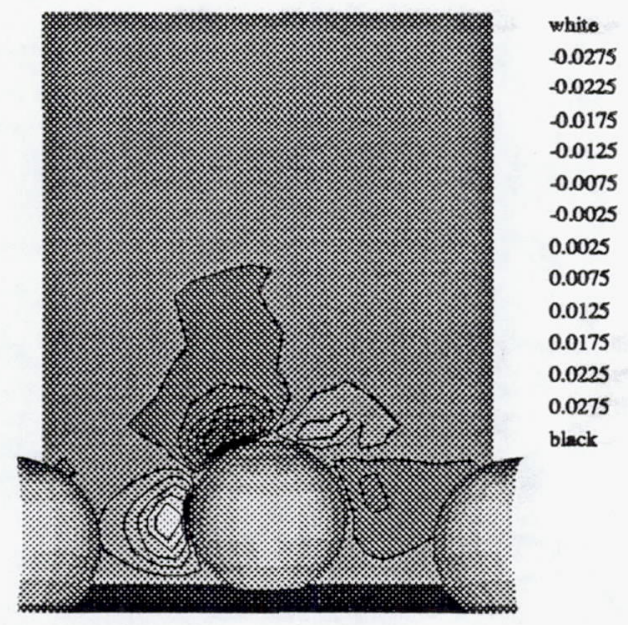

Figure 6: Side view of the vertical velocity contours on a standarddensity domain with a $5 X 5 X 5$ mesh along the vertical symmetry plane extending from the wall to the free stream.

gets forced upward (the wall prevents it from going down).

Reverse-flow regions indicate the importance of inertia since no reverse-flow regions exist in a viscous dominated flow. Thus, the upward and upstream velocity in the wake indicates that a chimney vortex, albeit a weak one, forms. Does the horseshoe vortex appear? With an isolated sphere, the horseshoe vortex begins in the stagnation region and extends into the wake. In distributed roughness, however, other roughness elements disturb the velocity in the wake; consequently, the stagnation region becomes the best place to evince the horseshoe vortex. Unfortunately, only an extremely weak horseshoe vortex exists. A true horseshoe vortex would appear in the stagnation region as a downward velocity near the element's centerline, an upstream velocity near the wall, and an upward velocity out in front of the element. As shown in figure 6, the downward velocity occurs near the sphere; however, neither the upward nor upstream velocities are evident.

The same velocity components on a horizontal plane passing through the center of the spheres are shown in figures 7 and 8. A high streamwise velocity "channel" appears between the rows of spheres as a consequence of forcing the flow along the geometric symmetry plane, and a reverse-flow region appears downstream of each element. The vertical velocity contours show downward flow ahead and 


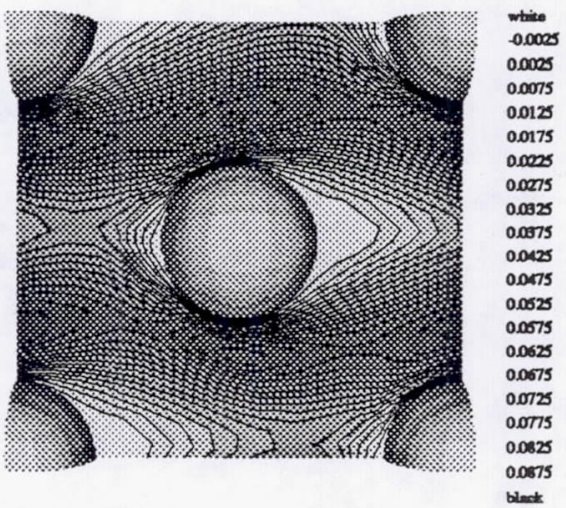

Figure 7: Plan view of the streamwise velocity contours on a standard-density domain with a $5 X 5 X 5$ mesh along a horizontal plane passing through the center of the spheres.

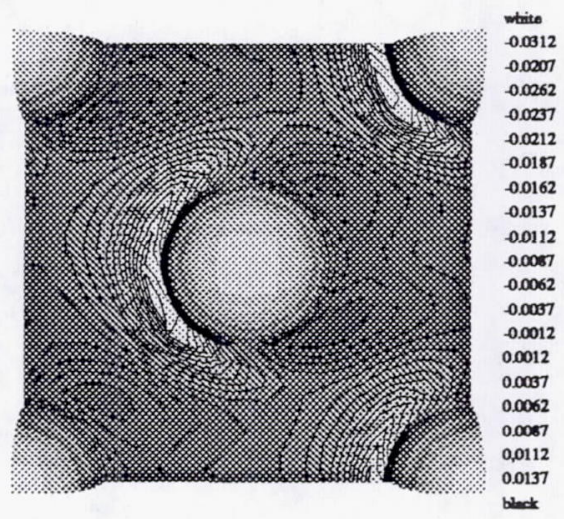

Figure 8: Plan view of the vertical velocity contours on a standarddensity domain with a $5 X 5 X 5$ mesh along a horizontal plane passing through the center of the spheres. 


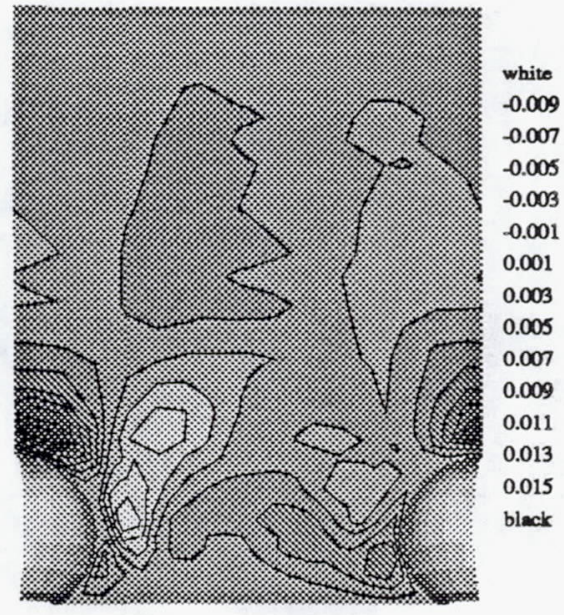

Figure 9: Cross-sectional view of the vertical velocity contours on a standard-density domain with a $5 X 5 X 5$ mesh along a vertical plane passing through the domain's inlet extending from the wall to the free stream.

upward flow behind each element, consistent with the continuity argument previously outlined.

Figures 9 and 10 present the vertical and spanwise velocity on a cross-sectional plane along the inlet of the domain. This plane cuts through the center of the corner spheres and lies just downstream of the center sphere in an imaginary cell upstream of the computational cell. Hence, this plane shows the central sphere's wake structure. The spanwise velocity always points toward the centerline, clearly showing how the fluid fills in the region behind the center sphere while being pushed inward by the corner spheres-both effects act in the same direction, yielding a strong spanwise velocity. The vertical velocity pattern illicits more interest. The central region shows upward velocity consistent with the continuity constraint discussed previously. A larger upward velocity region develops above each sphere-a manifestation of the inertia of the fluid passing over the obstacle. (If the flow were free of inertia, all velocity on this plane would be in the streamwise direction.) The two downward velocity regions reflect wake filling from above the corner spheres.

Streamwise vorticity on a cross-sectional plane at the inlet is shown in figure 11 . These vorticity regions seem to match those produced by the horseshoe and chimney vortices behind isolated rough- 


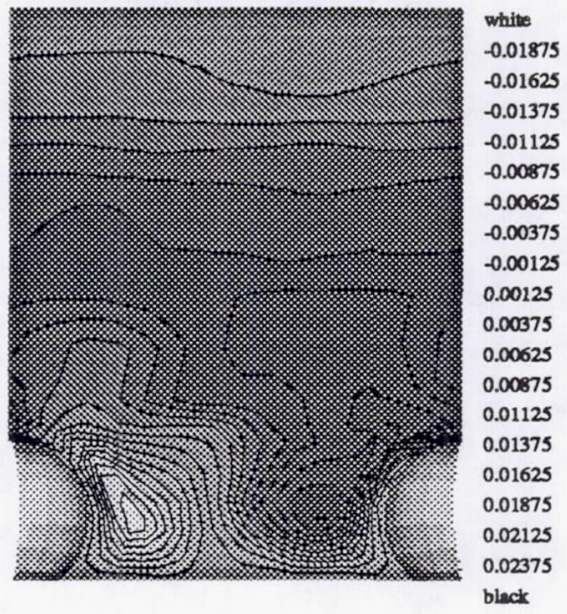

Figure 10: Cross-sectional view of the spanwise velocity contours on a standard-density domain with a $5 X 5 X 5$ mesh along a vertical plane passing through the domain's inlet extending from the wall to the free stream.

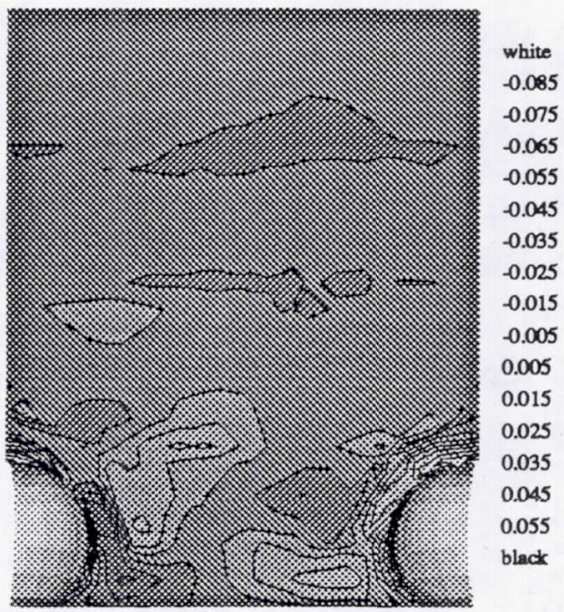

Figure 11: Cross-sectional view of the streamwise vorticity contours on a standard-density domain with a $5 X 5 X 5$ mesh along a vertical plane passing through the domain's inlet extending from the wall to the free stream. 


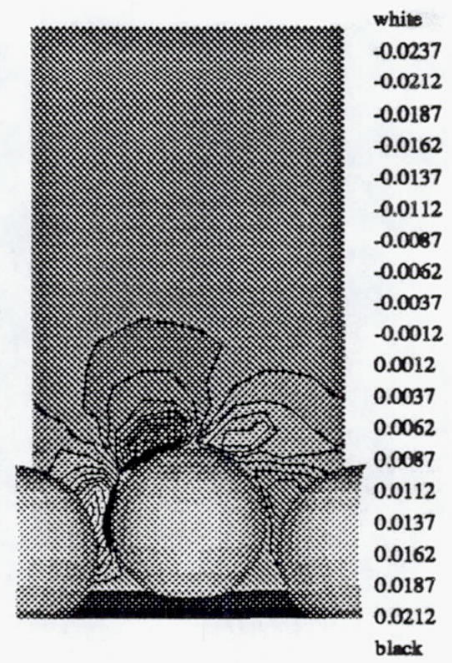

Figure 12: Side view of the vertical velocity contours on a highdensity domain with a $5 X 5 X 5$ mesh along the vertical symmetry plane extending from the wall to the free stream.

ness elements. Congruent with the vorticity system downstream of the center sphere without neighbors, on the left we see positive vorticity below and negative vorticity above the midpoint; the signs reverse on the right. We could easily link the trailing vortices from the sphere upstream and the vorticity pattern on the inlet plane. However, if these regions actually contain vortices, circular velocities should appear in the spanwise and vertical contour plots. We see no evidence of circular motion.

\subsection{Velocity contours high and low density domains}

The vertical velocity contours on the symmetry plane (see figures 12 and 13 ) show that the dense-packing maximum velocity decreases and the sparse-packing value increases compared to the standard domain. Evidently the sparse array allows an increase in momentum between the wake of one sphere and the stagnation region of the next. Further confirmation appears in the streamwise velocity contours on the horizontal plane passing through the sphere centers (not shown). The maximum streamwise velocity equals 0.0875 in the standard domain, 0.054 in the dense domain, and 0.115 in the sparse domain, a difference of a factor of two between the dense 


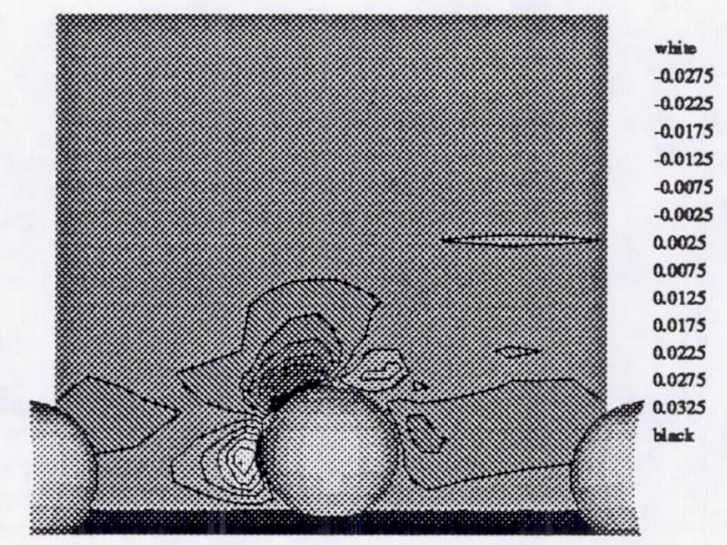

Figure 13: Side view of the vertical velocity contours on a low-density domain with a $5 X 5 X 5$ mesh along the vertical symmetry plane extending from the wall to the free stream.

and sparse cases. Clearly the dense array creates a large region of low-momentum fluid amid the spheres. An even more striking effect is evident in the spanwise and vertical velocity contours on the inlet cross-sectional plane (figures 14 and 15). Figure 14 shows the spanwise velocity contours for the dense array. The usual convergent velocity regions are shown; however, an outward velocity below this region exists, a downward velocity between these regions appears, and an upward velocity outside the downward velocity region develops - the horseshoe vortex. There is no evidence of the chimney vortex. Somehow the close proximity of the corner spheres to the wake of the center sphere generated the horseshoe vortex.

\section{Time Dependent Results}

During each run, we store streamwise, spanwise, and vertical velocity signals from five numerical probes. Probe locations are given in figures 16 and 17. Probe number 48, located behind the center sphere, lies within one sphere elevation from the wall; probe numbers 62,78 , and 88 lie approximately one and one-half sphere diameters from the wall; and probe number 120 lies two and one-half diameters above the wall. Probe numbers 62 and 78 occupy symmetric positions behind each of the two forward corner spheres. Similarly, probes 78 and 88 occupy equivalent positions behind the center sphere and one 


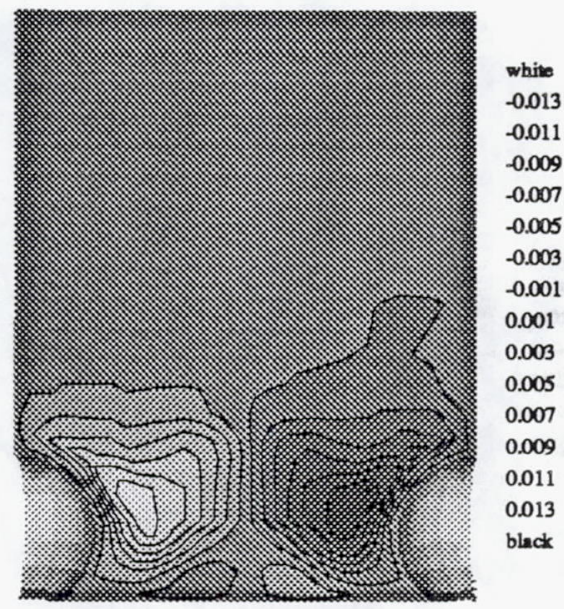

Figure 14: Cross-sectional view of the spanwise velocity contours on a high-density domain with a $5 X 5 X 5$ mesh along a vertical plane passing through the domain's inlet extending from the wall to the free stream.

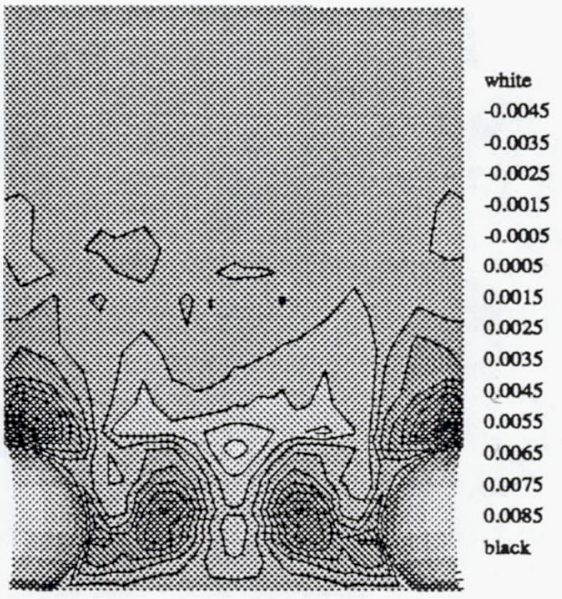

Figure 15: Cross-sectional view of the vertical velocity contours on a high-density domain with a $5 X 5 X 5$ mesh along a vertical plane passing through the domain's inlet extending from the wall to the free stream. 
i20

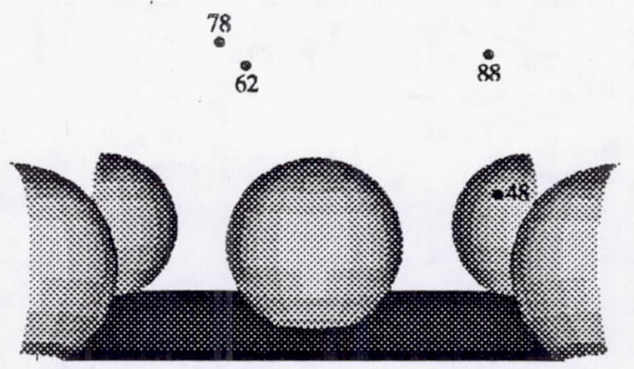

Figure 16: Side view of the probe locations.

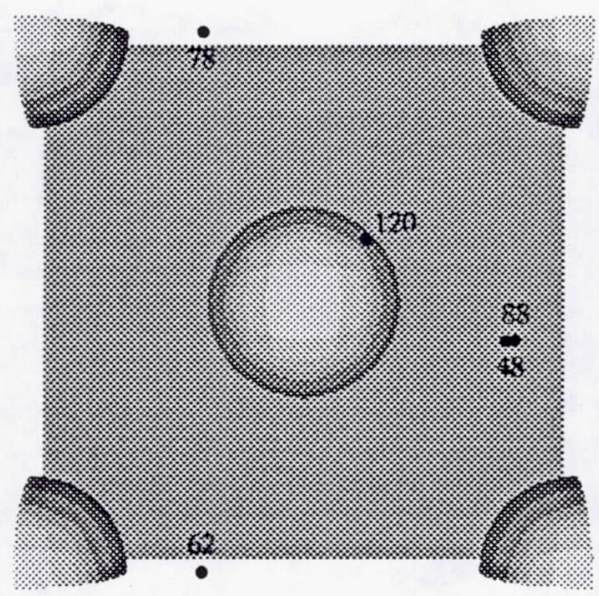

Figure 17: Plan view of the probe locations. 

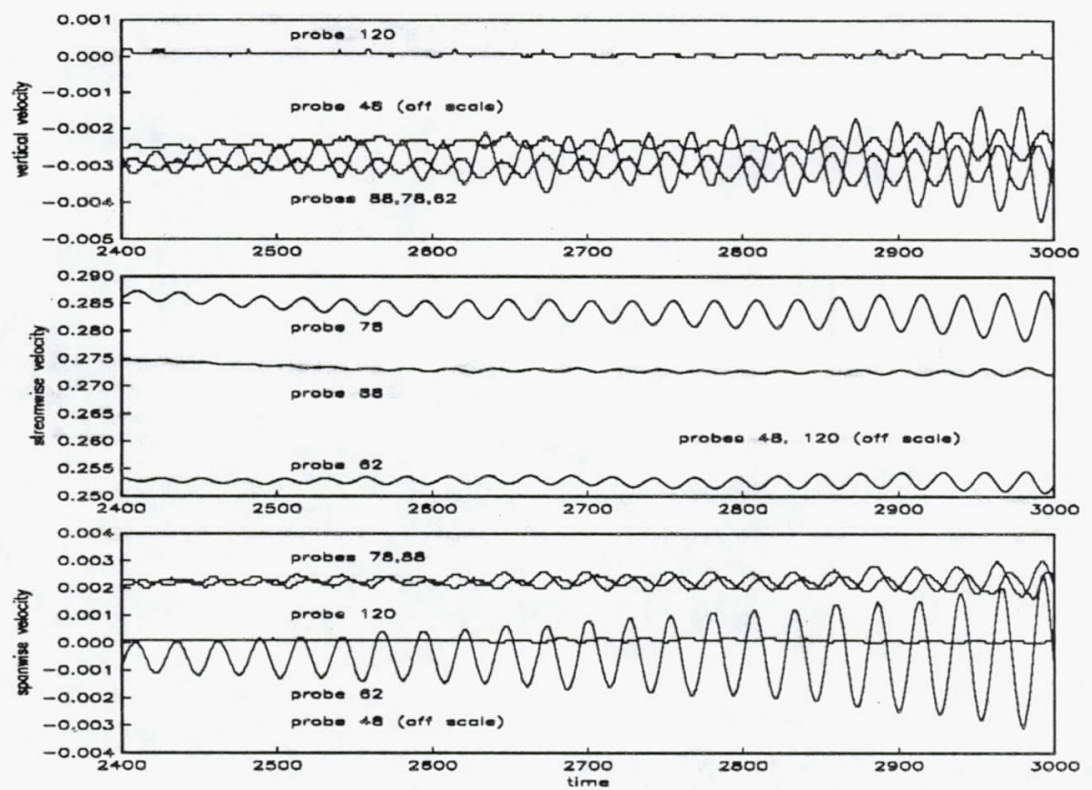

Figure 18: Vertical (top figure), streamwise (middle figure), and spanwise (bottom figure) velocity versus time for the five probes in the standard-density domain.

of the forward corner spheres. Thus, differences in the signals from each of these probes may reveal flow asymmetries and phase shifts in the disturbance waves.

\subsection{Standard density domain}

Using the standard domain, mesh dependent solutions revealed both steady and unsteady time-asymptotic states. Unsteady flows using a $5 X 5 X 5$ mesh decayed with a $7 X 7 X 7$ mesh.

Results using a $5 X 5 X 5$ mesh are given in figure 18. Although not shown, the total kinetic energy variation (an indication of flow development toward the time-asymptotic state) was less than 0.1 percent over the time contained in the graphs. This corresponds to a dimensionless convection time, $\tau_{c}=\Delta t U_{\infty} / L_{x}$, of 66 , or a dimensionless diffusion time, $\tau_{d}=\Delta t \nu / k^{2}$, equal to 0.51 . These numbers indicate that a fluid element in the free stream passed across the domain 66 times, but momentum only diffused across approximately $0.7 k$ during the measuring interval. The small change in total kinetic energy, however, indicates that the flow may appropriately classified 

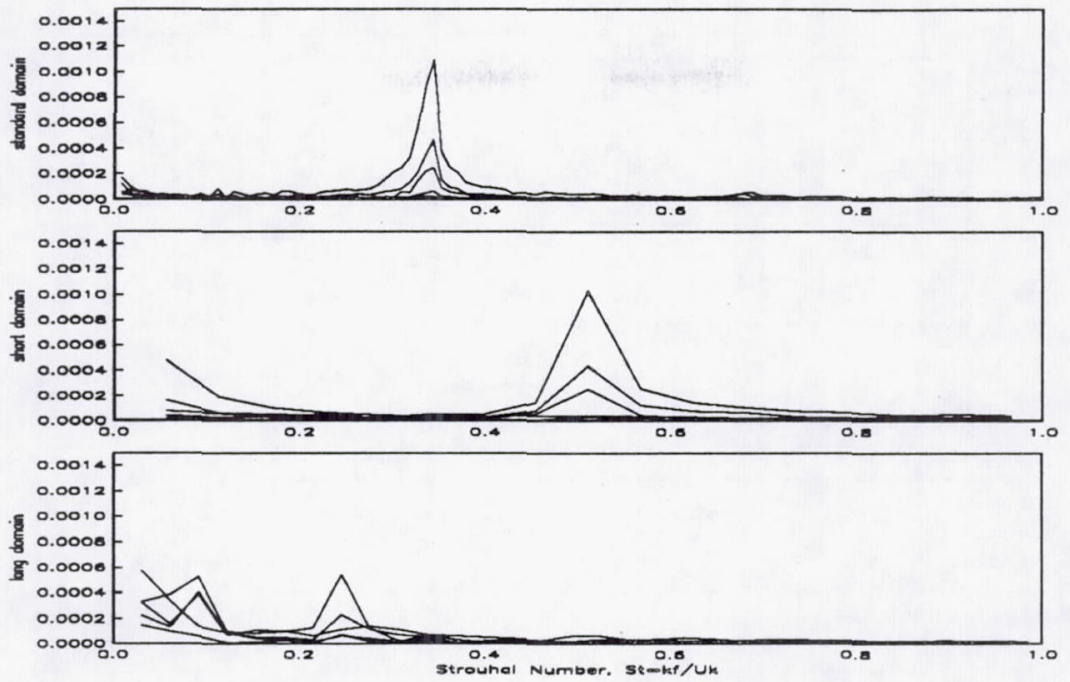

Figure 19: Vertical velocity amplitude for each of the five probes versus Strouhal number in the standard- (top figure), high- (middle figure), and low-density (bottom figure) domains.

as time-asymptotic. No oscillations developed in any of the velocity components from probe numbers 48 and 120 until the r.m.s. value of probe 78 's streamwise velocity reached 0.5 percent of the free-stream velocity. At this point probe 120 began a low-level oscillation. Vertical, streamwise, and spanwise oscillations with a $180^{\circ}$ phase shift occurred in probes 78 and 88 ; a small phase shift exists between probes 62 and 88 . These oscillations produce a strong single spike at $S t_{k}=k f / U_{k}=0.33$ in the standard domain's frequency spectrum (see figure 19). This corresponds to the "passing" frequency, $U_{k} / L_{x}$, for the time a parcel of fluid at elevation $y=k$ requires to cross between spheres spaced at a distance $L_{x}$ with a Blasius velocity $U_{k}$.

\subsection{High and low density domains}

If the roughness density controls the characteristic frequency, a lower oscillation frequency would exist in a low-density domain, and a higher one in a high-density domain. The high-density domain's streamwise length equals 6.0 ; the low-density domain's streamwise length equals 12.0. These lengths correspond to passing frequency 


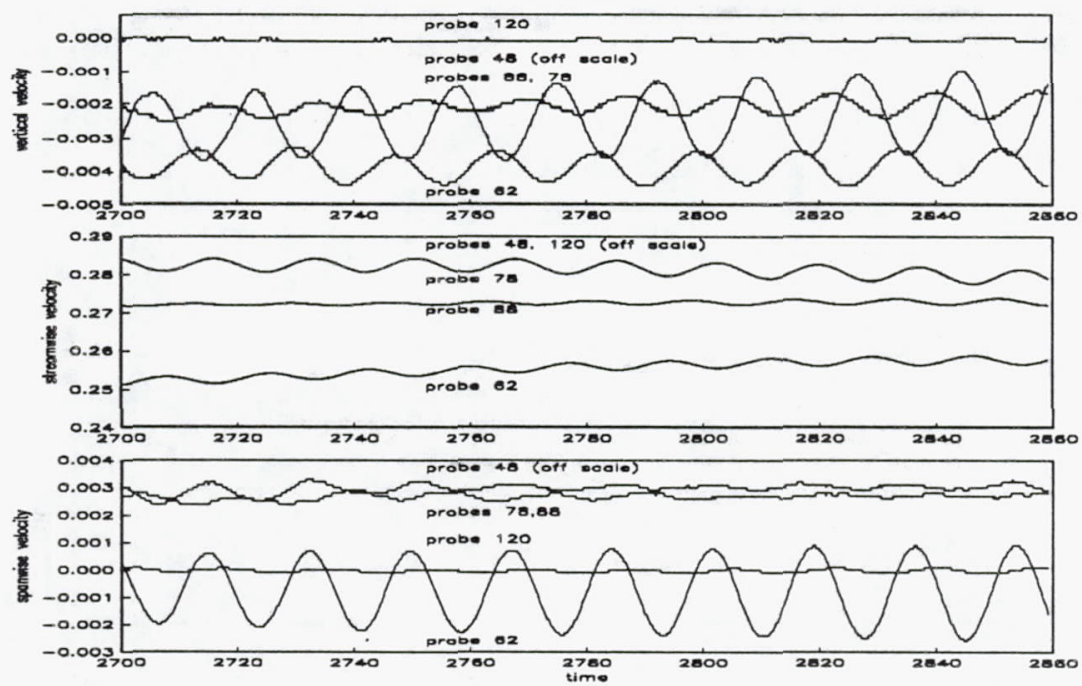

Figure 20: Vertical (top figure), streamwise (middle figure), and spanwise (bottom figure) velocity versus time for the five probes in the high-density domain.

based Strouhal numbers of 0.47 and 0.23 for the high- and low-density domains respectively. The time-dependent data from the five probes are given in figures 20 and 21 for these cases. Although not obvious from these figures, the dominant oscillation frequency, according to the frequency spectra shown in figure 19, does agree with the predicted Strouhal numbers given above.

\section{Analysis}

\subsection{Mean flow variations with roughness density}

At first glance it would appear that the displacement and momentum thicknesses would increase as the roughness elements become more densely packed. However, in the limit of infinite roughness density, these quantities would equal their Blasius values since the roughness effectively becomes a flat plate. A plot of $\delta^{*}$ and $\theta$ versus roughness density would show Blasius values at both the infinitedensity and zero-density limits. In between, the displacement thickness increases and the momentum thickness decreases compared to 


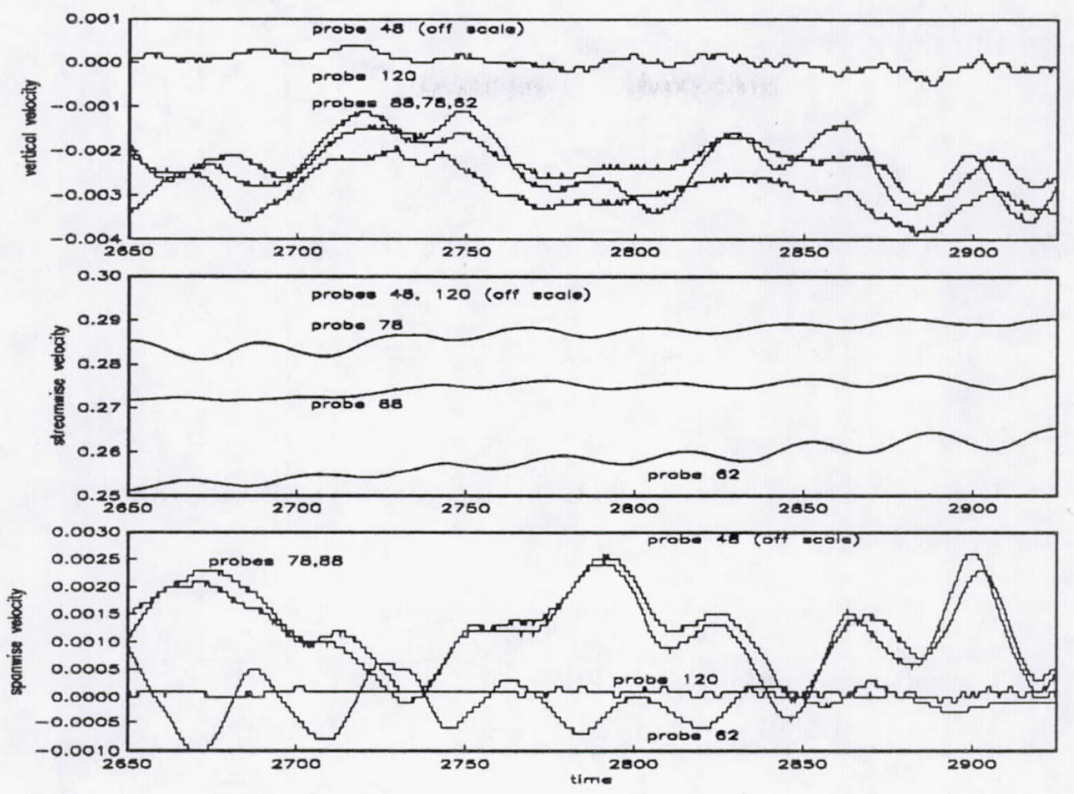

Figure 21: Vertical (top figure), streamwise (middle figure), and spanwise (bottom figure) velocity versus time for the five probes in the low-density domain.

Blasius values, consistent with inflected or adverse pressure gradient profiles.

Another parameter which changes with roughness density is the friction coefficient. Schlichting investigated how the flow resistance depends on roughness density [Schlichting, 1936]. He defined roughness density as

$$
F_{r}=\frac{\text { projected area of roughness on plate }}{\text { plate area }} .
$$

and found that the maximum resistance does not occur at the maximum roughness density but at a considerably lower value:

$$
\left(F_{r}\right)_{\max }=0.4
$$

The three roughness densities used in this study correspond to $F_{r}=$ 0.20 in the standard domain, $F_{r}=0.28$ in the high-density domain, and $F_{r}=0.14$ in the low-density domain. Schlichting defines a resistance coefficient in terms of the additional drag due solely to the roughness elements as $W_{r}$ and the corresponding friction coefficient 
due to the roughness elements as

$$
\left(C_{f}\right)_{\text {rough only }}=\frac{W_{r}}{\frac{1}{2} \rho U_{k}^{2} F_{r}} .
$$

Defining the ratio of rough- to smooth-wall friction factors as $\chi$ gives

$$
\left(C_{f}\right)_{\text {rough only }}=\frac{\left(C_{f}\right)_{\text {smooth }}(\chi-1)}{F_{r}} .
$$

Using the given values of $F_{r}$ and the results from table 1 for $\chi$ gives the results shown in table 2 . Also included are Schlichting's experimental results.

\begin{tabular}{|c|c|c|c|}
\hline Case & $F_{r}$ & $U_{k} / U_{\infty}$ & $\left(C_{f}\right)_{\text {rough only }}$ \\
\hline standard-density & 0.20 & 0.43 & 0.019 \\
high-density & 0.28 & 0.43 & 0.013 \\
low-density & 0.14 & 0.43 & 0.023 \\
Schlichting & 0.126 & 0.704 & 0.014 \\
Schlichting & 0.349 & 0.678 & 0.017 \\
Schlichting & 0.907 & 0.826 & 0.007 \\
Schlichting & 0.126 & 0.570 & 0.011 \\
\hline
\end{tabular}

Table 2: Roughness friction coefficient versus roughness density, comparison between numerical results and experiments.

Although the smallest total friction factor occurs in the lowdensity domain, we see that the friction factor due to the spheres alone is greatest in this case. The low-density domain permits more acceleration between spheres resulting in a faster approaching stream; consequently, each element produces greater resistance.

The numerical cases studied laminar flow and yielded roughness resistance coefficients higher than Schlichting's turbulent-flow results. This comports with expected behavior: an increase in friction coefficient with decreasing Reynolds number.

\subsection{Rough to smooth wall comparison}

The previous discussion concerns the effect of roughness density on the rough-wall boundary-layer integral quantities; this section relates the roughness quantities to the corresponding Blasius or 
smooth-wall values. All cases reveal a larger dimensionless displacement thickness than the Blasius value 1.72, a dimensionless momentum thickness smaller than the Blasius value 0.664, and a shape factor larger than the Blasius value 2.59. These results reflect the nature of the roughness boundary layer: a low-momentum region surrounding the roughness elements and a high-momentum region just above the roughness. Evidently, the spheres push fluid toward the free stream, leaving the region within one diameter from the wall with low momentum. Near the wall, the term $\left(1-U / U_{\infty}\right)$ is large, yielding a larger $\delta^{*}$. Also near the wall, $U / U_{\infty}$ is small, yielding a lower $\theta$.

The difference between the rough- and smooth-wall displacement thicknesses indicates the extent of the velocity profile displacement due to roughness. For the smcoth wall we can estimate the displacement thickness as $\delta^{*} \approx \delta / 3=3.67$; a typical rough-wall measured value gives $\delta^{*} \approx 3.90$. Therefore, the additional displacement due to roughness normalized by the roughness height equals $\Delta \delta^{*} / k=(3.90-3.67) / 2.8=0.082$. Comparing this measured change in displacement thickness to the volumetric average thickness of the roughness itself,

$$
\frac{\Delta h}{k}=\frac{2\left(\frac{4}{3} \pi R^{3}\right)}{k L_{x} L_{z}}=0.11,
$$

shows that, roughness displaces the Blasius layer an additional three quarters of the average roughness height, $\Delta \delta^{*} / \Delta h \approx 0.75$.

\subsection{Streamwise vorticity}

The experiments with isolated roughness show the importance of trailing vortices on boundary-layer instabilities. With this in mind, it is of interest to examine the distributed roughness flow for streamwise vortices. With the aid of figures 9 and 10, the spanwise and vertical velocity contours show no evidence of circular flow. Instead, the vorticity patterns depend on the gradients of mean velocity. Mathematically, the streamwise vorticity component equals

$$
\zeta_{x}=-(\partial w / \partial y-\partial v / \partial z)
$$

In the results with spherical roughness, the variation of spanwise velocity with vertical elevation, $\partial w / \partial y$, dominates the other term. Referring to figure 10 , we see that $w$ grows in magnitude from zero 
at the wall to a maximum at the sphere's midplane and back to zero above two diameters. On the right side of the plane, $w$ is positive; hence, $\partial w / \partial y>0$ near the wall, and the vorticity is negative. Above the midplane, $\partial w / \partial y<0$, and the vorticity is positive. The opposite signs correspond to the left half of the plane. This pattern exactly matches the streamwise vorticity regions of figure 11. Evidently, the presence of other spheres and the relatively low Reynolds number used in the computations prevent the strong streamwise vortices observed in experiments with isolated roughness.

\subsection{Oscillation frequency and phase}

The instability scenario begins when the local recirculation regions behind each sphere become unsteady above a certain critical Reynolds number (still unknown). This unsteady motion affects the mean flow by introducing pressure and velocity disturbances which, due to the periodic boundary conditions and incompressibility of the fluid, instantly fill the entire domain.

Once introduced, these disturbances grow or decay while propagating downstream with the local convection velocity of the mean flow. Eventually, those disturbances receiving the most energy in unstable modes dominate the flow. It appears that growth does not occur in the recirculation regions behind each sphere, since there is little incentive for amplification this close to the wall. Instead, the major growth occurs in the destabilized region just above the inflection point.

This comports with the stability characteristics governed by $U^{\prime \prime}(y)$. Inflected profiles usually have a higher amplification rate for the same frequency and Reynolds number than a profile with purely negative $U^{\prime \prime}(y)$ [Obremski \& Others, 1969]. Although inflected profiles are less stable, there is a stabilizing effect in the region between the inflection point and wall where $U^{\prime \prime}(y)>0$. An examination of the streamwise momentum equation reveals that $U^{\prime \prime}(y)$ acts like a force per unit mass on a parcel of fluid. When positive, as within the region between the wall and the inflection point, it accelerates or stabilizes a fluid parcel; outside of the inflection point it opposes or destabilizes the motion. This explains why oscillations at probe 48 , located below the inflection point, are small; why oscillations at probes 62,78 , and 88 , located just above the inflection point, are 
large; and why oscillations at probe 120 , located in a region where $U^{\prime \prime}(y)$ is small, are small. What explains the oscillation frequency?

Apparently, the most amplified disturbances occur in the layer just above the spheres, presumably carried downstream with a velocity of the local mean flow. Upon reaching the exit boundary, the disturbances reappear along the inlet boundary then resume their journey downstream. Once again, they encounter the exit boundary, and the pattern repeats ad infinitum. A disturbance interacts with the low-momentum recirculation zones behind a sphere each time it passes overhead. (It also interacts with higher momentum regions, but these regions do not respond significantly.) If the flow is unstable, the interaction increases the amplitude of the disturbance and causes the recirculation zone to move more vigorously. This generates a periodic motion with period equal to the time for a fluid element to pass between spheres at the elevation of the most amplified disturbance-in this case the period equals $L_{x} / U_{k}$. An individual recirculation region interprets this as a periodic forcing or "buffeting." If this buffeting frequency falls near the body's natural frequency, a response at the buffeting frequency occurs. This is referred to as the "lock-in" mode [Karniadakis \& Triantafyllou, 1989]. When the buffeting frequency differs greatly from the natural frequency, the flow responds at the isolated-body natural frequency, referred to as "non-lock-in." Evidently, the three roughness density cases under consideration-standard, high, and low-all satisfy the lock-in mode criteria. In each case, the oscillation frequency equals the forcing frequency.

Presumably, a higher or lower density case would show the nonlock-in mode. In this case, the oscillation frequency would equal the isolated element natural frequency and would be independent of periodic boundary conditions or element spacing. We may also expect a shear-layer based instability instead of one based on vortex shedding.

According to Karniadikis and Triantafyllou, 1989, the disturbance or forcing amplitude also influences the response. They present a plot of forcing amplitude versus forcing frequency, with unsteady regions labeled "lock-in" and "non-lock-in." The lock-in region occurs within an open-end-up parabolic curve centered at the natural frequency of the isolated body. The non-lock-in region surrounds the lock-in zone. Furthermore, the unsteady region-whether lock-in or non-lock-in-only occurs above a threshold amplitude. Thus, forcing 
at the natural frequency with an amplitude just above the threshold will produce a lock-in response; forcing with the same amplitude at a slightly different frequency will produce a non-lock-in response. As the forcing amplitude increases, the lock-in mode region grows; presumably, at infinite amplitude any forcing frequency will yield the lock-in behavior.

In the numerical calculations shown here, the only forcing is due to numerical approximations, and it seems reasonable to assume that the forcing level is greater with a $5 X 5 X 5$ mesh than with a $7 X 7 X 7$ mesh. Therefore, the lock-in region is larger with the $5 X 5 X 5$ mesh (possibly the lack of any response with the $7 X 7 X 7$ mesh indicates that the disturbance level with a $7 X 7 X 7$ mesh falls below the threshold amplitude). We conclude that the unsteady results will always depend on the forcing amplitude or mesh resolution in flows with no externally applied disturbances.

We've discused the oscillation amplitude and frequency, can we learn anything from the phase between different probes? In the highdensity domain (see figure 20 ), probes 62,78 , and 88 oscillate in all three directions while probes 48 and 120 show weak or no oscillations. In all directions, probes 62 and 78 oscillate with approximately a $180^{\circ}$ phase shift, and probes 78 and 88 oscillate with a $90^{\circ}$ phase shift. The $90^{\circ}$ phase shift occurs at a frequency of 0.054 cycles per time corresponding to a time shift of 4.6 units between peaks. The streamwise distance between probes 78 and 88 equals approximately 3.0 length units in the high-density domain; therefore, the streamwise phase speed equals

$$
C_{\phi}=\frac{3.0}{4.6}=0.65 \text {. }
$$

The Blasius velocity at the elevation of these probes equals 0.62 , further evidence that the disturbances propagate with the local convection velocity. A disturbance with this frequency and phase speed corresponds to a wavelength of

$$
\lambda=\frac{C_{\phi}}{f}=12.1 .
$$

This wavelength equals twice the domain length, and the $90^{\circ}$ phase shift between probes 3.0 length units apart corresponds to one quarter of a wave with a wavelength of 12.0 length units.

How can a disturbance with wavelength equal to twice the domain length exist in a domain with periodic boundary conditions? 
As in all numerical simulations, disturbances must satisfy the boundary conditions. This may be a restriction in one-dimensional computations; however, periodic boundaries may not restrict the disturbance character in two- or three-dimensional domains when the disturbances display only narrow spanwise or vertical extent. When a small disturbance travels downstream at an angle oblique with respect to the direction of periodic boundary conditions, or when it rises toward the free stream while moving downstream, there's no need for the disturbance to match inlet boundary conditions upon reaching the exit-it moved relative to its original position. Hence, the disturbance wavelength may be longer or shorter than the domain's length. Evidently, this is what occurs with small roughness elements.

The oscillations change in the low-density domain: probes 48 and 120 oscillate more vigorously than in the high-density domain, and the phase shifts between different probes no longer remain equal in the three directions (see figure 21). In the vertical direction, the phase shift between probes 88 and 78 approximately equals $5^{\circ}$ (corresponding to 4 time units); the phase shift between probes 62 and 78 equals $10^{\circ}$ (corresponding to 8 time units). Although probe 48's results are not shown, probes 48 and 120 oscillate nearly in-phase with a $5^{\circ}$ to $10^{\circ}$ phase shift from the other three. A zero phase shift exists between probes 62,78 , and 88 in the streamwise direction. In the spanwise direction, a small $5^{\circ}$ phase shift exists between probes 78 and 88 and nearly a $180^{\circ}$ phase shift between these probes and probe 62 . Clearly the simple structure evident in the high-density case is lost when the spheres are spaced further apart.

Can we classify the various waves appearing in the different cases? Instabilities in periodic domains involve either standing or traveling waves. Since the standing wave's phase speed equals zero (allowing independence of frequency and wavelength) a flow field dominated by a single standing wave reveals signals from probes located at different points with either a $0^{\circ}$ or $180^{\circ}$ phase shift. On the other hand, a traveling wave must must satisfy $C_{\phi}=f \lambda$, where the frequency equals $f$ and the wavelength equals $\lambda$. In this case, any phase shift between probes is possible.

With the periodic pattern of spheres, the recirculation zone behind each element at a Reynolds number below that associated with vortex shedding contains a standing wave: the entire separation zone oscillates at a single frequency. This motion influences those particles 
outside the recirculation zone, but, since they fall in a region dominated by convection, the standing-wave description would not apply, and the flow contains a traveling wave. A probe, however, measures the same frequency within the recirculation zone or outside in the convection zone; although, the two signals differ in phase-phase speed equals zero within the recirculation zone, and is finite outside.

The vortex-shedding mode at higher velocity contains no standing waves. In this regime, shed vortices convect downstream, and numerical probes located at different positions in the wake measure a phase shift between signals corresponding to the time for shed structures to pass.

In the high-density domain the $180^{\circ}$ phase shift between probes 62 and 78 indicate a standing wave in the horizontal direction, while the $90^{\circ}$ shift between probes 78 and 88 indicates a traveling wave in the streamwise direction.

In the low-density domain nearly all the signals from the various probes show small phase shifts corresponding to traveling waves. Only the spanwise signal from probes 62 and 88 show the characteristic $180^{\circ}$ phase shift associated with standing waves. The non-zero but small phase shifts correspond to a rapid traveling wave; the $180^{\circ}$ shift may indicate a standing wave or a traveling wave with wavelength equal to the domain length.

Apparently, the high-density domain's disturbances behave like standing waves, and the low-density domain's disturbances behave more like traveling waves. This indicates a prominence of vortex shedding in the low-density domain and vortex waving in the highdensity domain.

\subsection{Two cell behavior}

What happens to the characteristic frequency after adding another cell to the domain? Two possibilities exist: the oscillation frequency drops by a factor of two, as a sub-harmonic oscillation with wavelength equal to twice the one-cell length occurs; or the frequency remains the same.

In the first scenario, the periodic boundary conditions must influence the flow field, changing the number of cells probably changes the solution, and the individual roughness elements do not directly produce disturbances leading to oscillations; instead, a shear layer 
based disturbance takes place. In the second scenario, it appears that each roughness element contributes to the disturbance, the oscillation seems directly linked to the spacing between elements, and no solution variations occur with changes in cell number.

Although the two-cell results are not shown, the two-cell highdensity domain yields $S t_{k}=0.49$, and the two-cell low-density domain yields $S t_{k}=0.24$. These agree with the one-cell results, and it appears that the single cell adequately accounts for the important physics. Furthermore, the various phase shifts between signals from different probes match in the one- and two-cell cases.

\subsection{Comparison with experiments}

Experiments [Tadjfar \& Others, 1985] at $R e_{k}=310$ and $k / \delta^{*}=$ 1.6 , with an array of spherical roughness elements similar to the standard density case used in this study, reveal $S t_{k}=f k / U_{k}=0.08$ using the undisturbed Blasius velocity and 0.23 using the measured velocity at the top of a sphere. These results compare favorably with $S t_{k}=0.23$ with isolated spheres [Mochizuki, 1961], but differ from the $S t_{k}=0.33$ numerical results. This disparity cannot be resolved without additional experimental results using a different roughness density. The numerical and experimental results agree in one area, however. The peak amplitude occurred near $1.5 k$ from the wall in both cases, although Tadjfar took no measurements within the roughness elements.

\section{Conclusions}

Since identical oscillation frequencies were obtained with periodic boundary conditions applied across both one- and two-cell domains, the domain length does not govern the oscillation frequency; instead, the primary instability associated with a periodic array of spheres protruding from a flat surface occurs at a frequency which depends on the streamwise spacing between elements and the local velocity at the elevation of the sphere's crest. This also means that the characteristic frequency remains independent of the isolated sphere shedding frequency, and any similarities between the two are merely coincidental. 
This oscillation frequency, called the passing frequency for the time required for a fluid particle to pass from one sphere to the next, may also be thought of as a forcing frequency due to disturbances generated in the wake region behind the individual spheres. The response where the oscillation frequency equals the forcing frequency is called a lock-in. In the lock-in mode, the frequency of oscillation must lie near the shedding frequency of a similar body located in an infinite domain - in this case, the similar body equals a single sphere attached to a flat surface.

At $R e_{k}=175$, no streamwise vortices based on the flow's inertia exist; instead, the flow field contains regions of streamwise vorticity explained entirely by continuity considerations.

\section{References}

Corke, T. C., Bar-Sever, A. \& Morkovin, M. V. 1986. Experiments on transition enhancement by distributed roughness. Physics of Fluids, 29(10).

Dryden, H. L. 1953. Review of published data on the effect of roughness on transition from laminar to turbulent flow. Journal of the Aeronautical Sciences, 20(7).

Gartshore, I. \& de Croos, K. 1979. Equilibrium boundary layers over very rough surfaces. In $A G A R D$ Conference Proceedings No.271, Turbulent Boundary Layers Experiments, Theory and Modelling.

Gregory, N. \& Walker, W. S. 1951. The effect on transition of isolated surface excrescences in the boundary layer. Technical 
Report 2779, ARC R and M.

Gupta, A. K. 1980. Some observations in the wake of a small vertical cylinder attached to a flat plate. Physics of Fluids, 23(1).

Karniadakis, G. E., Mikik, B. B. \& Patera, A. T. 1988. Minimum dissipation transport enhancement by flow destabilization: Reynolds analogy revisited. Journal of Fluid Mechanics, $192,365-391$.

Karniadakis, G. E. \& Triantafyllou, G. S. 1989. Frequency selection and asymptotic states in laminar wakes. Journal of Fluid Mechanics, 199, 441-469.

Kendall, J. M. 1981. Laminar boundary-layer velocity distortion by surface roughness: Effect upon stability. Paper 81-0195, AIAA.

Laurien, E. \& Kleiser, L. 1989. Numerical simulation of boundarylayer transition and transition control. Journal of Fluid Mechanics, 199, 403-440.

Lee, B. \& Soliman, B. 1977. An investigation of the forces on threedimensional bluff bodies in rough-wall turbulent boundary layers. Journal of Fluid Engineering, 99, 503-510.

Mason, P. J. \& Morton, B. R. 1987. Trailing vortices in the wakes of surface-mounted obstacles. Journal of Fluid Mechanics, 175, $247-293$.

Mason, P. J. \& Sykes, R. I. 1979. Three-dimensional numerical integrations of the navier-stokes equations for flow over surfacemounted obstacles. Journal of Fluid Mechanics, 91, 433-450.

Mochizuki, M. 1961. Smoke observations on boundary-layer transition caused by a spherical roughness element. Journal of the Physical Society of Japan, 16(5).

Morkovin, M. V. 1989. Guide to experiments on instability and laminar-turbulent transition in shear layers. Cleveland, Ohio: NASA Lewis Research Center.

Obremski, H. J., Morkovin, M. V. \& Landahl, M. 1969. A portfolio of stability characteristics of incompressible boundary layers. AGARDograph 134, AGARD. 
Orszag, S. A. \& Kells, L. C. 1980. Transition to turbulence in plane Poiseuille and plane Couette flow. Journal of Fluid Mechanics, 96.

Reshotko, E. \& Leventhal, L. 1981. Preliminary experimental study of disturbances in a laminar boundary-layer due to distributed surface roughness. Paper 81-1224, AIAA.

Schlichting, H. 1936. Experimental investigation of the problem of surface roughness. Technical Memorandum 823, NACA.

Sedney, R. 1973. A survey of the effects of small protuberances on bounary-layer flows. AIAA Journal, 11(6).

Singer, B., Reed, H. L., \& Ferziger, J. H. 1986. Investigation of the effects of initial disturbances on plane channel transition. Paper 86-0433, AIAA.

Spalart, P. R. \& Yang, K. 1987. Numerical study of ribbon-induced transition in blasius flow. Journal of Fluid Mechanics, 178, $345-365$.

Tadjfar, M. 1990. Receptivity of a laminar bounary layer to the interaction of a three-dimensional roughness element with timeharmonic free-stream disturbances. $\mathrm{PhD}$ thesis, The Ohio State University.

Tadjfar, M. E., Reshotko, E., Dybbs, A. \& Edwards, R. V. 1985. Velocity measurements within boundary-layer roughness using index matching. FED Publication 33, ASME. 
Public reporting burden for this collection of information is estimated to average 1 hour per response, including the time for reviewing instructions, searching existing data sources, gathering and maintaining the data needed, and completing and reviewing the collection of information. Send comments regarding this burden estimate or any other aspect of this Davis Highway, Suite 1204, Arlington, VA 22202-4302, and to the Office of Management and Budget, Paperwork Reduction Project (0704-0188), Washington, DC 20503.

1. AGENCY USE ONLY (Leave blank)

2. REPORT DATE

August 1992

3. REPORT TYPE AND DATES COVERED

4. TITLE AND SUBTITLE

Technical Memorandum

5. FUNDING NUMBERS

Direct Numerical Simulation of Instabilities in Parallel Flow With

Spherical Roughness Elements

\section{AUTHOR(S)}

\section{R.G. De Anna}

WU-505-62-OK

1L161102AH45

\section{PERFORMING ORGANIZATION NAME(S) AND ADDRESS(ES)}

NASA Lewis Research Center

8. PERFORMING ORGANIZATION

Cleveland, Ohio 44135-3191 REPORT NUMBER

and

Propulsion Directorate

E-7291

U.S. Army Aviation Systems Command

Cleveland, Ohio 44135-3191

9. SPONSORING/MONITORING AGENCY NAMES(S) AND ADDRESS(ES)

National Aeronautics and Space Administration

Washington, D.C. 20546-0001

and

U.S. Army Aviation Systems Command

St. Louis, Mo. 63120-1798

10. SPONSORING/MONITORING

AGENCY REPORT NUMBER

NASA TM-105847

TR-92-C-023

\section{SUPPLEMENTARY NOTES}

R.G. De Anna, Propulsion Directorate, U.S. Army Aviation Systems Command. Responsible person, R.G. De Anna, (216) 433-3385.

12a. DISTRIBUTION/AVAILABILITY STATEMENT

12b. DISTRIBUTION CODE

Unclassified - Unlimited

Subject Category 34

\section{ABSTRACT (Maximum 200 words)}

Results from a direct numerical simulation of laminar flow over a flat surface with spherical roughness elements using a spectralelement method are given. The numerical simulation approximates roughness as a cellular pattern of identical spheres protruding from a smooth wall. Periodic boundary conditions on the domain's horizontal faces simulate an infinite array of roughness elements extending in the streamwise and spanwise directions, implies the parallel-flow assumption, and results in a closed domain. A body force, designed to yield the horizontal Blasius velocity in the absence of roughness, sustains the flow. Instabilities above a critical Reynolds number reveal negligible oscillations in the recirculation regions behind each sphere and in the free stream, high-amplitude oscillations in the layer directly above the spheres, and a mean profile with an inflection point near the sphere's crest. The inflection point yields an unstable layer above the roughness (where $\left.U^{\prime \prime}(y)<0\right)$ and a stable region within the roughness (where $\left.U^{\prime \prime}(y)>0\right)$. Evidently, the instability begins when the low-momentum or wake region behind an element, being the region most affected by disturbances (purely numerical in this case), goes unstable and moves. In incompressible flow with periodic boundaries, this motion sends disturbances to all regions of the domain. In the unstable layer just above the inflection point, the disturbances grow while being carried downstream with a propagation speed equal to the local mean velocity; they do not grow amid the low energy region near the roughness patch. The most amplified disturbance eventually arrives at the next roughness element downstream, perturbing its wake and inducing a global response at a frequency governed by the streamwise spacing between spheres and the mean velocity of the most amplified layer.

\section{SUBJECT TERMS}

Surface roughness; Transition; Boundary layer flow; Direct numerical simulation

15. NUMBER OF PAGES

36

16. PRICE CODE

$\mathrm{AO} 3$

17. SECURITY CLASSIFICATION OF REPORT

Unclassified

18. SECURITY CLASSIFICATION OF THIS PAGE

Unclassified
19. SECURITY CLASSIFICATION OF ABSTRACT

Unclassified 